J Rheumatol. 2015 July ; 42(7): 1194-1202. doi:10.3899/jrheum.141129.

\title{
YOGA IN SEDENTARY ADULTS WITH ARTHRITIS: EFFECTS OF A RANDOMIZED CONTROLLED PRAGMATIC TRIAL
}

\author{
Steffany Moonaz, Clifton O. Bingham III, Lawrence Wissow, and Susan J. Bartlett
}

\begin{abstract}
Objective-To evaluate the impact of Integral-based hatha yoga in sedentary people with arthritis.
\end{abstract}

Methods-75 sedentary adults aged 18+ with rheumatoid arthritis (RA) or knee osteoarthritis (OA) were randomly assigned to 8 weeks of yoga (2 60 min classes and 1 home practice/wk) or waitlist. Poses were modified for individual needs. The primary endpoint was physical health (SF36 Physical Component Summary [PCS]) adjusted for baseline; exploratory adjusted outcomes included fitness, mood, stress, self-efficacy, SF36 health-related quality of life (HRQL) and RA disease activity. In everyone completing yoga, we explored long-term effects at 9 months.

Results-Participants were mostly female (96\%), white (55\%), and college-educated (51\%), with a mean (SD) age of 52 (12). Average disease duration was 9 (9) yrs. and 49\% had RA. At 8 weeks, yoga was associated with significantly higher PCS $(6.5 ; 95 \%$ CI: 2.0,10.7), walking capacity $(125 \mathrm{~m}$; 95\% CI:15,235), positive affect $(5.2 ; 95 \%$ CI:1.4,8.9) and lower CES-D (-3.0; 95\% CI: -4.8,-1.3). Significant ( $<<.05)$ improvements were evident in SF36 Role Physical, Pain, General Health, Vitality and Mental Health scales. Balance, grip strength, and flexibility were similar between groups. 22/28 on waitlist completed yoga. Among all yoga participants, significant ( $\mathrm{p}<.05)$ improvements were observed in mean PCS, flexibility, 6-min walk, all psychological and most HRQL domains at 8 weeks with most still evident 9 months later. Of seven adverse events, none were associated with yoga.

Conclusions-Preliminary evidence suggests yoga classes may help sedentary individuals with arthritis safely increase physical activity and improve physical and psychological health, and HRQL.

\section{Key Indexing Terms}

yoga; rheumatoid arthritis; osteoarthritis; mobility; HRQL

For people with arthritis, physical activity is essential for optimal disease management and preserving mobility $(1,2)$. Exercise helps maintain range of motion, joint stability, and

Corresponding Author: Dr. Susan J. Bartlett, (514) 843-1465 (v), (514) 843-1493 (f), susan.bartlett@mcgill.ca.

For reprint requests: Dr. Susan J. Bartlett, Associate Professor of Medicine, McGill University (RVH), 687 Pine Avenue, Ross 4.31, Montreal, QC, H3A 1A1

Trial registration. Clinical Trials NCT00349869

Conflicts of Interest: S Moonaz provides continuing education training for yoga therapists to work with clients with arthritis. She also developed content for a DVD for the Arthritis Foundation entitled "Arthritis Friendly Yoga" based on this work. 
muscle mass, while reducing pain and fatigue (3-5). The stress reduction associated with exercise can help individuals adaptively cope to living with a painful, chronic illness, and emerging evidence also suggests beneficial effects on systemic inflammation and immune function $(6,7)$. However, despite the well-known benefits of physical activity, up to $44 \%$ of people with arthritis report no leisure time physical activity (8) and $76 \%$ are inadequately active (9). Indeed, arthritis appears to be a major barrier to physical activity; among people with heart disease, those with arthritis were $60 \%$ more likely to be inactive (10).

Yoga may be well-suited for arthritis by combining physical activity with potent stress management techniques including breathing, relaxation, and mindfulness (11). In 2012, 20 million US adults (nearly 1 in 10) practiced yoga to improve health and fitness, with $40 \%$ starting in the past year alone (12). The 2007 National Health Interview Survey listed yoga as the sixth most commonly used complementary health practice among adults (13). In both healthy and clinical populations, the health benefits of yoga appear to be similar to other forms of exercise (14-16). The strongest evidence of benefit is for reducing pain (standardized mean difference [SMD] $-.74 ; 95 \%$ confidence interval $[\mathrm{CI}]-.97$ to -.52 ), pain-related disability (SMD -.79 (95\% CI -1.02 to -.56$)(17)$ and improving mood (SMD $-.65 ; 95 \%$ CI -.89 to -.42 ) (18). However, medical professionals may be concerned that yoga's emphasis on changing positions and flexibility could stress vulnerable joints affected by arthritis.

Unfortunately, the evidence base is limited regarding yoga in arthritis. We summarized 10 peer reviewed articles and abstracts of yoga and arthritis through 2010 (19); the existing studies, conducted in diverse populations around the world were mostly of low quality (small samples, non-randomized trials, heterogeneity of dose, methods and outcomes) and precluded drawing conclusions. Other reviews of recent trials in rheumatic conditions have noted ongoing concerns with study quality, but the emerging evidence does suggest yoga may improve arthritis symptoms (pain, stiffness, tenderness), function, and mood (19-22). Given its popularity and availability, well-controlled trials are needed to guide recommendations about yoga in arthritis $(15,20-22)$.

Hence, our goal was to contribute evidence about outcomes associated with initiating yoga practice in sedentary people with arthritis. An 8-week program was developed, emphasizing individualized adaptations and monitoring, to promote successful transition to home practice. We hypothesized that yoga would improve physical health, fitness, psychological function, health related quality of life (HRQL) and arthritis self-efficacy, with no worsening of RA disease activity.

\section{Methods Study Design}

A parallel-arms pragmatic 8-week RCT compared Integral-based hatha yoga and waitlist groups. Pragmatic RCTs use rigorous methodology (randomization, blinding, allocation concealment) to reduce bias, but allow flexibility in intervention delivery and enhance external validity by testing existing programs in real-world settings and with less stringent inclusion criteria (23). Individuals were randomly assigned 1:1 to yoga or waitlist. This 
study was approved by the Johns Hopkins Institutional Review Board and registered with clinicaltrials.gov (NCT00349869).

The study was conceptualized for RA; after 31 patients were enrolled, additional funding was secured and the study was expanded to include knee OA. Although these diseases have different underlying mechanisms, both result in significant joint pain and disability, and community classes are likely to include people with both conditions. Additional secondary endpoints were added.

We also explored the effects of yoga over time. After completing 8 week follow-up assessments, waitlisted participants were invited to join upcoming classes. We also completed follow-up assessments on these participants after 8 weeks of yoga, and on everyone who had completed yoga 9 months after classes ended.

\section{Participants}

Eligibility criteria included: 1) RA age 18-70; OA 18+; 2) sedentary (physically active for 20 min < 3 times/wk); and 3) diagnosed with RA, OA or probable knee OA as indicated by a positive response to questions used in trials to identify knee OA (24). Exclusion criteria were: 1) use of cane, walker, or wheelchair; 2) other inflammatory conditions; or 3) surgery within 6 months. For RA, medical clearance was required. Eligibility criteria were deliberately broad to ensure participants reflected typical people with arthritis living in the community. Enrollment occurred from June 2005 through July 2008 and ended when 75 participants had been enrolled.

\section{Recruitment and allocation}

Participants were recruited from arthritis clinics, private practices, and using local flyers. The Maryland Arthritis Foundation Chapter also publicized the study in newsletters. Potential participants were screened by phone and eligible persons provided written consent and underwent baseline assessments. Rheumatologists and all assessors remained blinded to treatment assignment.

Simple random assignment by a third party using a web-based randomizer was used for allocation; allocation sequence was concealed from all study team members by using sequentially-numbered, sealed opaque envelopes which were opened by the coordinator only once baseline assessments were completed. Yoga classes began once 12-15 people were available (i.e., had been randomized after baseline and/or completed waitlist).

\section{Treatment arms}

Yoga consisted of 60-minute classes held twice weekly for 8 weeks at two hospital-affiliated fitness centers in Baltimore, MD. The program was designed by a registered yoga therapist (SM) with input from Johns Hopkins Arthritis Center faculty. Two yoga therapists with 10+ years of experience taught classes. Yoga therapists have additional training to address needs of people with diverse conditions/abilities.

Each class began with questions/comments ( $5 \mathrm{~min}$ ), breathing exercises and chanting ( 5 $\mathrm{min}$ ), warm-up and moving sequence (surya namaskara; $15 \mathrm{~min}$ ), and isometric poses 
(asanas) (20 min) to increase strength, flexibility and balance. Classes ended with deep relaxation (sivasana; $10 \mathrm{~min}$ ), a closing chant, and meditation (5 min). See supplemental file for sample class and modifications. Poses included gentle forward bends, backbends, twists, balances, standing, sitting and lying poses, and were modified for individual at the discretion of the teacher and/or participant. Complexity of poses and intensity was standardized to allow gradual progression. Eight weeks was selected for the intervention as this provides sufficient time to introduce independent practice and is a common duration of introductory classes. Props included blocks, straps, blankets, and chairs. Participants were encouraged to try new skills but remain safe and avoid discomfort. Written instructions with pictures for home practice and selected readings describing potential benefits of yoga components (breathing, meditation, mindfulness) were provided weekly. Home practice evolved gradually to develop the skills and confidence for long-term adherence. Participants were asked to keep arthritis medications constant and were queried regularly by coordinators about any changes.

The waitlist group received usual care for 8 weeks. They were asked to maintain current levels of physical activity and inform coordinators of changes in health or arthritis medications. After week 8 assessments, they were invited to participate in upcoming classes.

\section{Dependent Measures}

Measures were obtained at baseline, week 8 and 9 months (yoga only) at the Johns Hopkins Bayview General Clinical Research Center by blinded assessors.

The primary endpoint was the SF-36 Physical Component Summary (PCS) at week 8 . The SF-36 assesses eight domains with higher scores representing better HRQL. The PCS and Mental Component Scores ((MCS) differentially weight all scales using a t-score metric (mean 50 and standard deviation (SD) 10). The PCS, which emphasizes Physical Function, fulfilling Physical Roles and Bodily Pain, is reliable $(\mathrm{ICC}=0.81)$ and responsive to change $(\mathrm{SRM}=0.61)(25,26)$.

Exploratory endpoints included fitness, psychological function, and HRQL. Using standardized protocols, flexibility was measured using a sit-and-reach box (27), balance with one-leg stance (OLS; maximum 30s), and strength with a hand dynamometer (28). For each measure, the best of three attempts was recorded. The 6-min Walk (29) measured walking capacity.

The SF-36 MCS provided an overall index of mental health. Depressive symptoms were assessed using the 11-item CES-D(30), which has a similar factor structure and properties as the original scale and is validated in arthritis (31). The Positive and Negative Affect Scale (PANAS) assessed mood. The PANAS is reliable (r's 0.86 and 0.87 for PA and NA) and internally consistent (alpha $0.86-.90$ for PA and $0.84-0.87$ for NA) (32). Stress was measured with the Perceived Stress Scale (PSS) which is reliable (r's .84-.86) and correlates moderately with number and impact of life events (r's 0.33-0.49)(33). The Arthritis Self Efficacy Scale (ASES), which measures confidence to manage arthritis, is internally consistent ( $r=.94)$ and reliable ( $r$ 's $>0.85$ ) (34). For RA, a 28-tender and swollen joint count 
was conducted by trained assessors and participants completed the $100 \mathrm{~mm}$ Patient Global VAS. Attendance was recorded at classes.

\section{Statistical Analysis}

The study was designed and powered to detect an 8-point difference in PCS between groups. (The minimal clinically important difference for RA is 5). A sample size of 30/group was sufficient to detect this difference, assuming a SD of 11 points, power of $80 \%$, and significance of $5 \%$.

Groups were compared on sociodemographic and SF36 scores by arthritis type using t-tests and chi-square. To assess the impact of missing data for variables added after the study began, we compared characteristics of the first 31 participants with the last 44, and also used multiple imputation and last observation carried forward (LOCF).

The primary RCT analysis was ANCOVA by group with adjustment for baseline values (Model 1); in Model 2 we also added adjustment for age. Baseline covariates were chosen to improve the precision of estimates; age was explored given its associations with outcomes and due to chance imbalances between groups despite randomization (35) after first testing for homogeneity of regression slopes by treatment. Paired t-tests also explored within-group differences after 8 weeks of yoga and 9 months later.

RCT data were analyzed based on treatment assignment. Because this was an exploratory study, we did not control for multiple comparisons. All analyses were performed using IBM SPSS V21.

\section{Results}

Of 103 persons who contacted the research team, 19 were ineligible due to current physical activity (11), recent/planned surgery (5), use of assistive devices (3); 9 declined due to time requirements (see Figure 1). Thus, 75 participants were randomly assigned to yoga $(\mathrm{N}=40)$ or waitlist $(\mathrm{N}=35)$. Participants were mostly middle-aged (mean $\pm \mathrm{SD} ; 52 \pm 12$ yrs), female (96\%), and White (55\%), with 39\% Black. About half (51\%) were college-educated and $49 \%$ had RA with an average duration of $9 \pm 9$ yrs.

Of the last 44 enrolled, $67 \%$ had OA; the mean age of those enrolled when recruitment was expanded was significantly higher than the original cohort, but did not differ on other sociodemographic or arthritis variables [data not shown]. Yoga participants were significantly younger than waitlist, but did not otherwise differ (Table 1). RA participants were significantly younger and had significantly lower SF36 MCS, General Health, Social Function and Mental Health scores (Table 2).

Among 40 yoga participants, 7 withdrew (see Figure 1 for reasons) between baseline and the start of classes, and 8 withdrew during the intervention; thus, Week 8 data were available for 25 participants. Most who completed yoga (22; 79\%) attended at least 12/16 classes. Of 35 people waitlist, 7 withdrew; week 8 data were available for 28. Study completers did not differ significantly from withdrawals by age, sex, education, diagnosis, disease duration, 
pain or physical function, but were more likely to be minorities (OR 4.3; 95\% CI 1.5-12.4). The most common reasons for withdrawal were life events and scheduling.

\section{Physical health and fitness}

At week 8, in adjusted analyses (Model 1), the mean PCS score for yoga was 6.5 points higher than waitlist ( $\mathrm{p}<.001)$ (Table 3); this .7 SD difference is both statistically and clinically meaningful and persisted with adjustment for age (Model 2). Walking capacity was also significantly higher for yoga $(125 \mathrm{~m})$; there was a trend $(\mathrm{p}=.056)$ for sit and reach scores to also be higher. OLS and grip strength did not differ between groups. In Model 2, with additional adjustment for age, differences in flexibility reached statistical significance, however, 6-min walk, grip strength, and balance were not significantly different between groups. Results were similar using LOCF (data not shown).

\section{Psychological function}

At week 8, with adjustment for baseline, yoga reported significantly fewer depressive symptoms (CES-D) and higher positive affect (PANAS). However, groups did not differ on SF36 MCS, negative affect, perceived stress or arthritis self-efficacy. Results were unchanged with adjustment for age.

\section{HRQL}

At week 8, adjusted analyses showed yoga participants reported significantly less impairment on SF36 Role Physical, Body Pain, General Health, Vitality, and Mental Health scales, with trends ( $p<.08)$ evident in Physical Function and Role Emotional. Within groups, yoga improved significantly on all SF36 scales except Role Emotional, while scores were essentially unchanged in waitlist. Significant differences were evident in the same SF36 scales except General Health with further adjustment for age.

In 25 RA participants, we also explored changes in joint counts and Patient Global scores (Table 4). Swollen and tender joint counts decreased and Patient Global scores improved in both groups, and were not statistically different in baseline adjusted analyses between groups at week 8 .

\section{Yoga Group}

At week 8, 22 of 28 (79\%) waitlist participants began yoga. Characteristics were similar between waitlist participants who did and did not start yoga (data not shown). Overall, of 55 who began yoga, 44 (80\%) completed week 8 testing. PCS, all fitness (except OLS), psychological, and SF36 measures (except Role Emotional) improved significantly (Table 5). Follow-up data were available on 37 (67\%) 9 months after completing yoga. Improvements in PCS, sit-and-reach, 6-min walk, CES-D, positive and negative affect, perceived stress, and SF36 Physical Function, Role Physical, Bodily Pain, and Vitality scales were still evident.

\section{Adverse events}

No adverse events were specifically associated with yoga. The seven events (1 each) reported to the IRB for yoga (not attributed to yoga) included bruising (workplace accident), 
ovarian cancer recurrence, bladder cancer, tendinitis, hyperthyroidism, allergic reaction, and new RA diagnosis (study end). No adverse events occurred in the waitlist group.

\section{Discussion}

To date, this is the largest RCT of OA and RA, and the first to assess physical health and fitness using self-reported and performance measures along with psychological function and HRQL. This is only the second study in arthritis (of 20 published to date) to include safety data. As compared to waitlist, yoga was associated with substantial improvements in physical and general health perceptions, physical roles, walking, pain, energy, and mood. Regular yoga practice was not associated with worsening joint symptoms or adverse events. Indeed, in RA, swollen and tender joint counts decreased significantly with yoga, though a similar trend was evident in the waitlist.

Though 24\% dropped out of yoga, persistence was still higher than in many exercise programs (36), with most attending the majority of classes. The most common reason people withdrew was time/schedule conflicts. Others $(37,38)$ have also reported higher rates of adherence to yoga than studies with clinical populations where $<50 \%$ continue exercising by 3 months.(39) Reducing inactivity is an important public health challenge, especially in arthritis, where only 1 in 4 are meeting activity guidelines.(9) Notably, $80 \%$ of the waitlist group opted to take yoga. Assessments 9 months later indicated the durability of most improvements.

At week 8 , the 6.5 point difference between groups in PCS indicated important improvements in health. PCS scores reflect physical function, role physical (work and daily activity impairments due to physical health), pain, and energy. Indeed, in yoga, 16/44 (36\%) improved one category or more on the SF36 self-rating of health. We noted a trend toward improved physical function with yoga at week 8 , as have some $(37,40-42)$, though not all (43). When we pooled data from all yoga completers, there were significant improvements in all HRQL scales except Role Emotional which was already close to population norms at baseline.

We hypothesized that yoga would lead to improved flexibility, balance and strength. Flexibility, which has not been previously evaluated in arthritis, improved with practice, although differences were not statistically significant $(\mathrm{p}=.056)$. Since loss of joint mobility is common in arthritis, preserving range of motion is important for maintaining mobility. However, yoga was not associated with improved balance, in contrast to findings by others (44), perhaps because baseline values were already high. For example, $45 \%$ of participants scored at maximum levels (30s) at baseline; among those with scores $<30 \mathrm{~s}, 54 \%$ improved with yoga. T'ai Chi also may enhance balance and reduce the risk of falls in arthritis (45). Future yoga studies should further evaluate flexibility and balance. We also did not find improvements in grip strength; Garfinkel also found no change in grip strength with yoga in hand OA (46), although this has been reported by others in RA $(47,48)$. However, grip strength assesses upper limb strength and may not adequately characterize muscle strength of the entire body. Yoga targets many muscle groups, and many poses do not directly involve the upper limbs. 
Pain, one of the most widely studied outcomes in yoga, improved significantly with yoga. Some $(37,41,42,44,46)$ though not all $(42,43,49)$ have reported similar results. Yoga was associated with positive affect and fewer depressive symptoms, findings also reported by some $(37,40,44)$ though not all $(42,50)$. In contrast to Evans et al. (37), self-efficacy was not significantly different between groups, perhaps because both groups improved.

In RA, significant reductions in joint counts were observed in both groups. One other study reported reductions in joint counts (43); another found no change in C-reactive protein (CRP) with one week of yoga at a residential camp (49). Together, these results suggest that yoga is unlikely to worsen disease activity. Sensitive measures such as erythrocyte sedimentation rate, CRP and interleukin- 6 should be included in larger samples to evaluate the effect of yoga on systemic inflammation and immune reactivity.

A strength of this study was the controlled pragmatic study design. Randomization, blinding, concealment of treatment allocation, and use of flexible standardized protocols to guide progression increase confidence in our results. Including typical sedentary participants with RA and OA, evaluating classes specifically designed to promote independent practice, offering the program in community settings, and evaluating impact on HRQL increase the applicability and relevance of results. Although our program was developed by a multidisciplinary team (rheumatologists, psychologists, public health and exercise scientists) and tailored for arthritis, we used common hatha yoga poses and practices and a class length found in many introductory classes. Participants were taught to assess how they felt at each class and to adjust their practice accordingly. We also explored clinician-measured (fitness and clinical signs) and patient-centered (mood, stress, self-efficacy, symptoms) outcomes to gain insight into how yoga may impact health and well-being. Data collected on waitlist participants who later completed yoga supported the RCT findings, and many trends became statistically and clinically significant. We recently spoke with several participants who attended focus groups we later conducted on RA stiffness. Now (five years later), they commented that yoga had played a pivotal role in changing how they viewed their function, and capabilities and attitude toward living with RA; they credited yoga with helping them maintain a more active lifestyle.

Although this study provides preliminary evidence that yoga appears to be acceptable to people with arthritis and does not aggravate joint disease, caution is warranted. A longer period of practice might produce further gains, though potentially with an increased risk of injury. Despite improvements after 8 weeks, significant impairments were still evident in physical function, role physical, pain, general health, and energy. Classes were taught by experienced yoga therapists in community hospital fitness settings. Because classes began once $\sim 15$ participants were available, the length of time between baseline assessment and the start of yoga classes varied. We did not stratify by diagnosis, randomization did not result in group equivalence in age, and our sensitivity analyses used methods assuming data were missing at random, which we did not confirm. We cannot determine whether some yoga elements had more benefit than others, or the extent to which the yoga group experienced greater social support. Research to replicate and extend these findings across settings, instructors, and in diverse groups is ongoing. Inclusion of biomarkers may provide 
additional insight. Larger trials with active comparators are needed to establish the relative efficacy of yoga vs. traditional exercise and other mind-body practices.

In summary, this study contributes preliminary new evidence that sedentary individuals with RA and knee OA can safely learn to practice yoga in classes led by trained instructors who provide close supervision and individual attention. Eight weeks of classes and home practice was associated with clinically significant improvements in physical and mental health, fitness, psychological function, and HRQL with no adverse outcomes. Additional studies with active comparison groups in diverse settings and other forms of arthritis are necessary to support these findings and establish the benefits of yoga in relation to traditional exercise in people with arthritis.

\section{Supplementary Material}

Refer to Web version on PubMed Central for supplementary material.

\section{Acknowledgments}

The authors would like to acknowledge the assistance of Heather Keller, Devin Rand-Giovannetti, Chethan Kasargod, Isabel Roth, Michelle Jones, Uzma Haque, Jon Giles and Joan Bathon.

Support: National Center for Complementary and Alternative Medicine pilot project (Bartlett), National Institutes of Health pre-doctoral award 1F31AT003362-01A1 and Arthritis Foundation doctoral dissertation award (Moonaz)

\section{References}

1. American College of Rheumatology Subcommittee on Osteoarthritis Guidelines. Recommendations for the medical management of osteoarthritis of the hip and knee: 2000 update. Arthritis Rheum. 2000; 43:1905-15. [PubMed: 11014340]

2. American College of Rheumatology Subcommittee on Rheumatoid Arthritis Guidelines. Guidelines for the management of rheumatoid arthritis: 2002 update. Arthritis Rheum. 2002; 46:328-46. [PubMed: 11840435]

3. Fransen M, McConnell S. Exercise for osteoarthritis of the knee. Cochrane Database Syst Rev. 2008:CD004376. (Epub ahead of print). 10.1002/14651858.CD004376.pub2 [PubMed: 18843657]

4. Juhl C, Christensen R, Roos EM, Zhang W, Lund H. Impact of exercise type and dose on pain and disability in knee osteoarthritis: A systematic review and meta-regression analysis of randomized controlled trials. Arthritis Rheumatol. 2014; 66:622-36. [PubMed: 24574223]

5. Cramp F, Hewlett S, Almeida C, Kirwan JR, Choy EH, Chalder T, et al. Non-pharmacological interventions for fatigue in rheumatoid arthritis. Cochrane Database Syst Rev. 2013; 8:CD008322. [PubMed: 23975674]

6. Hurley MV, Mitchell HL, Walsh N. In osteoarthritis, the psychosocial benefits of exercise are as important as physiological improvements. Exercise and Sport Sciences Reviews. 2003; 31:138-43. [PubMed: 12882480]

7. Petersen AM, Pedersen BK. The anti-inflammatory effect of exercise. Journal of Applied Physiology. 2005; 98:1154-62. [PubMed: 15772055]

8. Centers for Disease Control and Prevention. State-specific prevalence of no leisure-time physical activity among adults with and without doctor-diagnosed arthritis--united states, 2009. MMWR. 2011; 60:1641-5. [PubMed: 22157882]

9. Hootman JM, Macera CA, Ham SA, Helmick CG, Sniezek JE. Physical activity levels among the general us adult population and in adults with and without arthritis. Arthritis Rheum. 2003; 49:129_ 35. [PubMed: 12579604] 
10. Centers for Disease Control and Prevention. Arthritis as a potential barrier to physical activity among adults with heart disease--united states, 2005 and 2007. MMWR. 2009; 58:165-9. [PubMed: 19247262]

11. Sharma M. Yoga as an alternative and complementary approach for stress management: A systematic review. Journal of Evidence-Based Complementary \& Alternative Medicine. 2014; 19:59-67. [PubMed: 24647380]

12. Yoga in america - 2012. [internet]. San Francisco, CA: Yoga Journal; 2012. [updated 2012; cited (Epub ahead of print)2014 Sep 1]. Available from: http://www.yogajournal.com/press/ yoga_in_america

13. Barnes PM, Bloom B, Nahin RL. Complementary and alternative medicine use among adults and children: United states, 2007. National Health Statistics Reports. 2008:1-23. Epub ahead of print. [PubMed: 19361005]

14. Raub JA. Psychophysiologic effects of hatha yoga on musculoskeletal and cardiopulmonary function: A literature review. Journal of Alternative and Complementary Medicine. 2002; 8:797812.

15. Bartlett SJ, Moonaz SH, Mill C, Bernatsky S, Bingham CO 3rd. Yoga in rheumatic diseases. Curr Rheumatol Rep. 2013; 15:387. [PubMed: 24173693]

16. Patel NK, Newstead AH, Ferrer RL. The effects of yoga on physical functioning and health related quality of life in older adults: A systematic review and meta-analysis. Journal of Alternative and Complementary Medicine. 2012; 18:902-17.

17. McCall MC, Ward A, Roberts NW, Heneghan C. Overview of systematic reviews: Yoga as a therapeutic intervention for adults with acute and chronic health conditions. Evidence-Based Complementary and Alternative Medicine. 2013; 2013:945895. [PubMed: 23762174]

18. Bussing A, Ostermann T, Ludtke R, Michalsen A. Effects of yoga interventions on pain and painassociated disability: A meta-analysis. J Pain. 2012; 13:1-9. [PubMed: 22178433]

19. Haaz S, Bartlett SJ. Yoga for arthritis: A scoping review. Rheum Dis Clin North Am. 2011; 37:3346. [PubMed: 21220084]

20. Uhlig T. Tai chi and yoga as complementary therapies in rheumatologic conditions. Best Practice \& Research: Clinical Rheumatology. 2012; 26:387-98. [PubMed: 22867933]

21. Sharma M. Yoga as an alternative and complementary approach for arthritis: A systematic review. Journal of Evidence-Based Complementary \& Alternative Medicine. 2014; 19:51-8. [PubMed: 24647379]

22. Cramer H, Lauche R, Langhorst J, Dobos G. Yoga for rheumatic diseases: A systematic review. Rheumatology (Oxford). 2013; 52:2025-30. [PubMed: 23934220]

23. Treweek S, Zwarenstein M. Making trials matter: Pragmatic and explanatory trials and the problem of applicability. Trials. 2009; 10:37. [PubMed: 19493350]

24. Altman R, Asch E, Bloch D, Bole G, Borenstein D, Brandt K, et al. Development of criteria for the classification and reporting of osteoarthritis. Classification of osteoarthritis of the knee. Diagnostic and therapeutic criteria committee of the american rheumatism association. Arthritis Rheum. 1986; 29:1039-49. [PubMed: 3741515]

25. Ware, JE., Jr; Kosinski, M.; Keller, SD. Sf-36 physical \& mental health summary scales: A user's manual. Boston, MA: The Health Institute, New England Medical Center; 1994.

26. Hurst NP, Ruta DA, Kind P. Comparison of the mos short form-12 (sf12) health status questionnaire with the sf36 in patients with rheumatoid arthritis. Br J Rheumatol. 1998; 37:862-9. [PubMed: 9734677]

27. Wells KF, Dillon EK. The sit and reach: A test of back and leg flexibility. Research Quarterly for Exercise \& Sport. 1952; 23:115-8.

28. Mathiowetz V, Weber K, Volland G, Kashman N. Reliability and validity of grip and pinch strength evaluations. J Hand Surg Am. 1984; 9:222-6. [PubMed: 6715829]

29. ATS Committee on Proficiency Standards for Clinical Pulmonary Function Laboratories. . Ats statement: Guidelines for the six-minute walk test. American Journal of Respiratory and Critical Care Medicine. 2002; 166:111-7. [PubMed: 12091180] 
30. Kohout FJ, Berkman LF, Evans DA, Cornoni-Huntley J. Two shorter forms of the ces-d (center for epidemiological studies depression) depression symptoms index. J Aging Health. 1993; 5:179-93. [PubMed: 10125443]

31. Rhee SH, Petroski GF, Parker JC, Smarr KL, Wright GE, Multon KD, et al. A confirmatory factor analysis of the center for epidemiologic studies depression scale in rheumatoid arthritis patients: Additional evidence for a four-factor model. Arthritis Care Res. 1999; 12:392-400. [PubMed: 11081010]

32. Watson D, Clark LA, Tellegen A. Development and validation of brief measures of positive and negative affect: The panas scales. J Pers Soc Psychol. 1988; 54:1063-70. [PubMed: 3397865]

33. Cohen, S.; Williamson, G. Perceived stress in a probability sample of the united states. In: Spacapam, S.; Oskamp, S., editors. The social psychology of health: Claremont symposium on applied social psychology. Newbury Park, CA: Sage; 1988.

34. Lorig K, Chastain RL, Ung E, Shoor S, Holman HR. Development and evaluation of a scale to measure perceived self-efficacy in people with arthritis. Arthritis Rheum. 1989; 32:37-44. [PubMed: 2912463]

35. Yu LM, Chan AW, Hopewell S, Deeks JJ, Altman DG. Reporting on covariate adjustment in randomised controlled trials before and after revision of the 2001 consort statement: A literature review. Trials. 2010; 11:59. [PubMed: 20482769]

36. Dishman, R. Determinants of participation in physical activity. In: Bouchard, C.; Shepard, R.; Stephens, T.; Sutton, J.; McPherson, B., editors. Exercise, fitness, and health. Champaign: Human Kinetics; 1990. p. 75-102.

37. Evans S, Moieni M, Lung K, Tsao J, Sternlieb B, Taylor M, et al. Impact of iyengar yoga on quality of life in young women with rheumatoid arthritis. Clin J Pain. 2013; 29:988-97. [PubMed: 23370082]

38. Flegal KE, Kishiyama S, Zajdel D, Haas M, Oken BS. Adherence to yoga and exercise interventions in a 6-month clinical trial. BMC Complement AlternMed. 2007; 7:37.

39. Woodard CM, Berry MJ. Enhancing adherence to prescribed exercise: Structured behavioral interventions in clinical exercise programs. J Cardiopulm Rehabil. 2001; 21:201-9. [PubMed: 11508179]

40. Kolasinski SL, Garfinkel M, Tsai AG, Matz W, Van Dyke A, Schumacher HR. Iyengar yoga for treating symptoms of osteoarthritis of the knees: A pilot study. J Altern Complement Med. 2005; 11:689-93. [PubMed: 16131293]

41. Ebnezar J, Nagarathna R, Yogitha B, Nagendra HR. Effects of an integrated approach of hatha yoga therapy on functional disability, pain, and flexibility in osteoarthritis of the knee joint: A randomized controlled study. J Altern Complement Med. 2012; 18:463-72. [PubMed: 22537508]

42. Park J, McCaffrey R. Chair yoga: Benefits for community-dwelling older adults with osteoarthritis. J Gerontol Nurs. 2012; 38:12-22. quiz 4-5. [PubMed: 22533347]

43. Badsha H, Chhabra V, Leibman C, Mofti A, Kong KO. The benefits of yoga for rheumatoid arthritis: Results of a preliminary, structured 8-week program. Rheumatol Int. 2009; 29:1417-21. [PubMed: 19184028]

44. Bosch PR, Traustadottir T, Howard P, Matt KS. Functional and physiological effects of yoga in women with rheumatoid arthritis: A pilot study. Altern Ther Health Med. 2009; 15:24-31. [PubMed: 19623830]

45. Logghe IH, Verhagen AP, Rademaker AC, Bierma-Zeinstra SM, van Rossum E, Faber MJ, et al. The effects of tai chi on fall prevention, fear of falling and balance in older people: A metaanalysis. Prev Med. 2010; 51:222-7. [PubMed: 20558197]

46. Garfinkel MS, Schumacher HR Jr, Husain A, Levy M, Reshetar RA. Evaluation of a yoga based regimen for treatment of osteoarthritis of the hands. J Rheumatol. 1994; 21:2341-3. [PubMed: 7699639]

47. Haslock I, Monro R, Nagarathna R, Nagendra HR, Raghuram NV. Measuring the effects of yoga in rheumatoid arthritis. Br J Rheumatol. 1994; 33:787-8. [PubMed: 8055212]

48. Dash M, Telles S. Improvement in hand grip strength in normal volunteers and rheumatoid arthritis patients following yoga training. Indian J Physiol Pharmacol. 2001; 45:355-60. [PubMed: 11881576] 
49. Telles S, Naveen KV, Gaur V, Balkrishna A. Effect of one week of yoga on function and severity in rheumatoid arthritis. BMC Res Notes. 2011; 4:118. [PubMed: 21481278]

50. Taibi DM, Vitiello MV. A pilot study of gentle yoga for sleep disturbance in women with osteoarthritis. Sleep Med. 2011; 12:512-7. [PubMed: 21489869] 


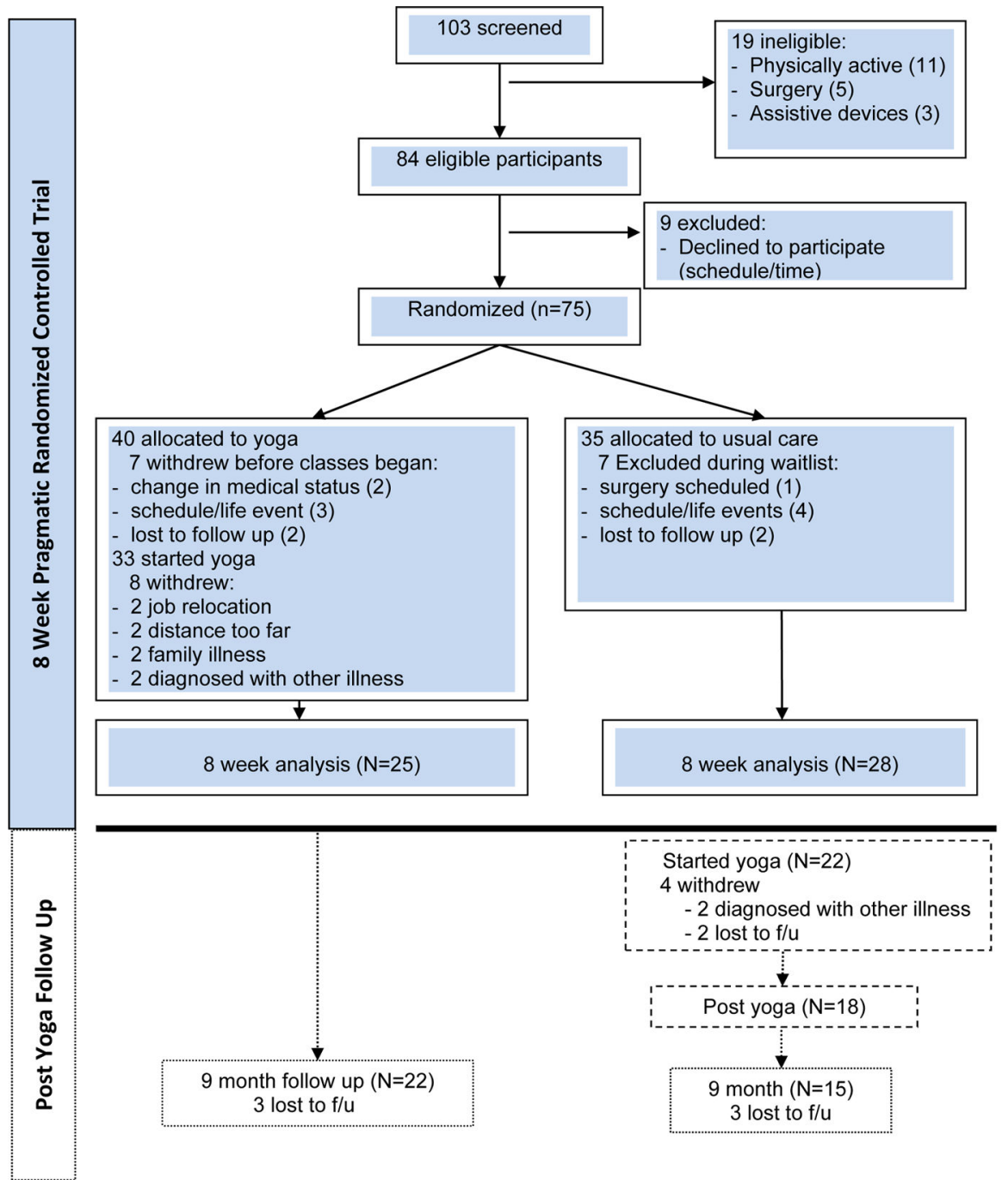

Figure 1.

Flow of yoga and usual care participants throughout 8 week randomized controlled trial and 9 month follow-up (yoga participants only). 


\section{Table 1}

Baseline characteristics of participants.

\begin{tabular}{lcc}
\hline Value & $\begin{array}{c}\text { Yoga } \\
\mathbf{N = 4 0}\end{array}$ & $\begin{array}{c}\text { Waitlist } \\
\mathbf{N = \mathbf { 3 5 }}\end{array}$ \\
\hline Age (mean \pm SD; yrs) & $49.2 \pm 13.2$ & $55.9 \pm 8.9$ \\
Female (\%) & $40(100)$ & $32(91)$ \\
Race (\%) & & \\
$\quad$ White & 57.5 & 51.4 \\
$\quad$ Black & 37.5 & 40.0 \\
Other & 5.0 & 8.6 \\
College graduate (\%) & 47.5 & 55.9 \\
Diagnosis (\% RA) & 55 & 43 \\
Disease duration (mean \pm SD; yrs) & $9.9 \pm 8.7$ & $8.6 \pm 9.4$ \\
\hline
\end{tabular}

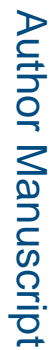

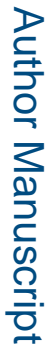

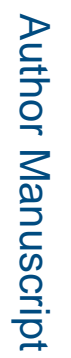




\section{Table 2}

Participant characteristics by arthritis type.

\begin{tabular}{lccc}
\hline Variable & $\begin{array}{c}\text { RA } \\
\mathbf{N = 3 7}\end{array}$ & $\begin{array}{c}\mathbf{O A} \\
\mathbf{N = 3 8}\end{array}$ & p-value \\
\hline Age & $46.6 \pm 12.1$ & $57.9 \pm 8.7$ & $<.001$ \\
Female sex (n, \%) & $36(97 \%)$ & $36(95 \%)$ & .572 \\
College graduate & $20(54 \%)$ & $19(50 \%)$ & .831 \\
Minority & $14(38 \%)$ & $20(53 \%)$ & .198 \\
Disease duration & $8.5 \pm 7.6$ & $10.1 \pm 10.2$ & .431 \\
SF36 Component Scores & & & \\
$\quad$ Physical Component (PCS) & $34.1 \pm 10.9$ & $35.5 \pm 9.8$ & .568 \\
$\quad$ Mental Component (MCS) & $48.6 \pm 12.4$ & $54.4 \pm 9.9$ & .032 \\
SF36 Scales & & & \\
$\quad$ Physical Function & $57.7 \pm 24.6$ & $49.6 \pm 21.5$ & .142 \\
Role Physical & $34.1 \pm 38.4$ & $43.4 \pm 37.5$ & .305 \\
Bodily Pain & $48.0 \pm 19.1$ & $53.7 \pm 17.4$ & .193 \\
$\quad$ General Health & $46.7 \pm 24.3$ & $66.7 \pm 18.2$ & .000 \\
Vitality & $45.6 \pm 21.2$ & $51.1 \pm 20.2$ & .272 \\
Social Function & $62.9 \pm 24.7$ & $79.6 \pm 22.6$ & .004 \\
Role Emotional & $68.7 \pm 42.4$ & $71.1 \pm 40.4$ & .811 \\
Mental Health & $69.7 \pm 21.1$ & $80.1 \pm 13.1$ & .014 \\
\hline
\end{tabular}

Values are the mean and SD unless otherwise indicated. 


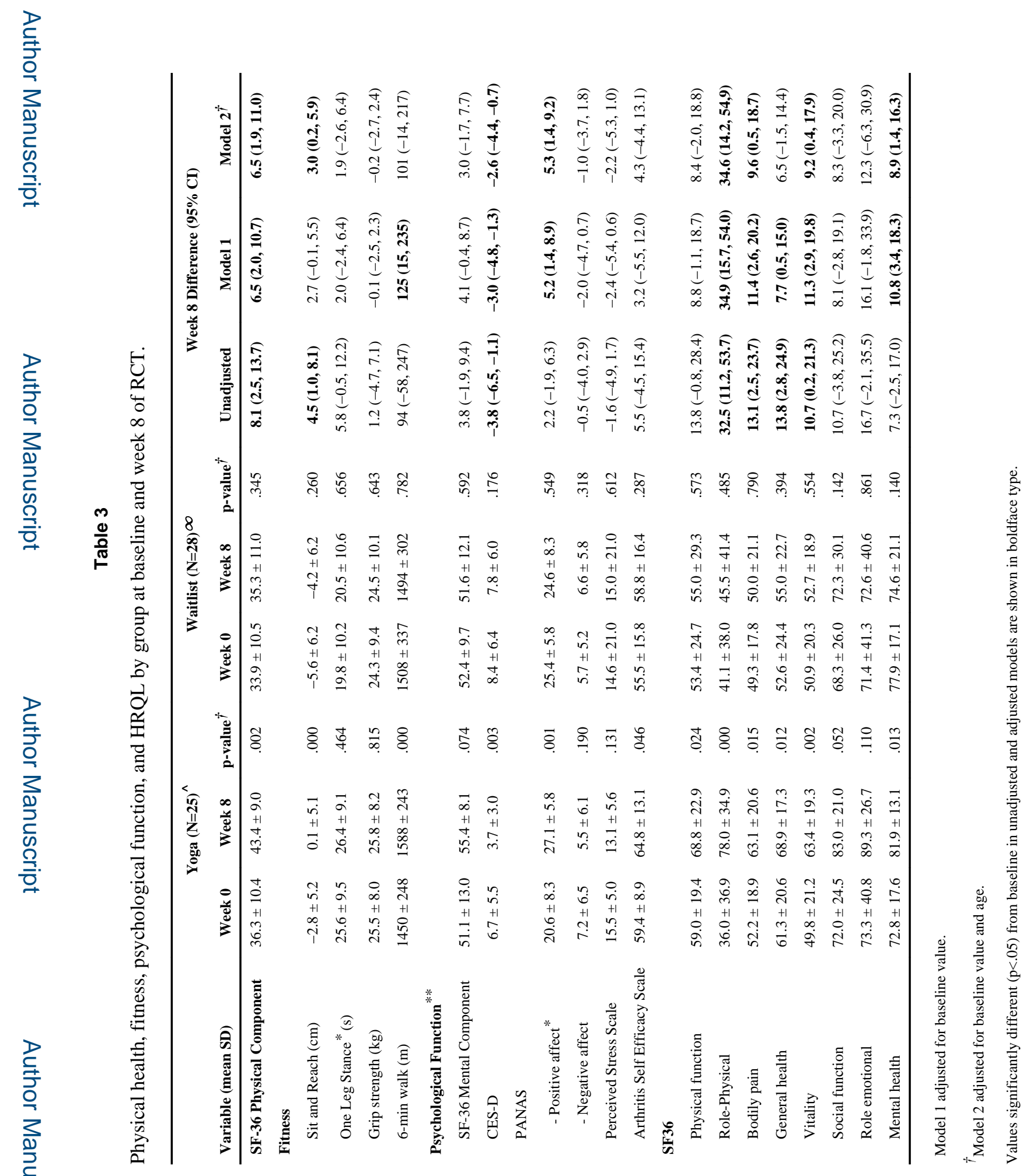

J Rheumatol. Author manuscript; available in PMC 2015 July 03. 


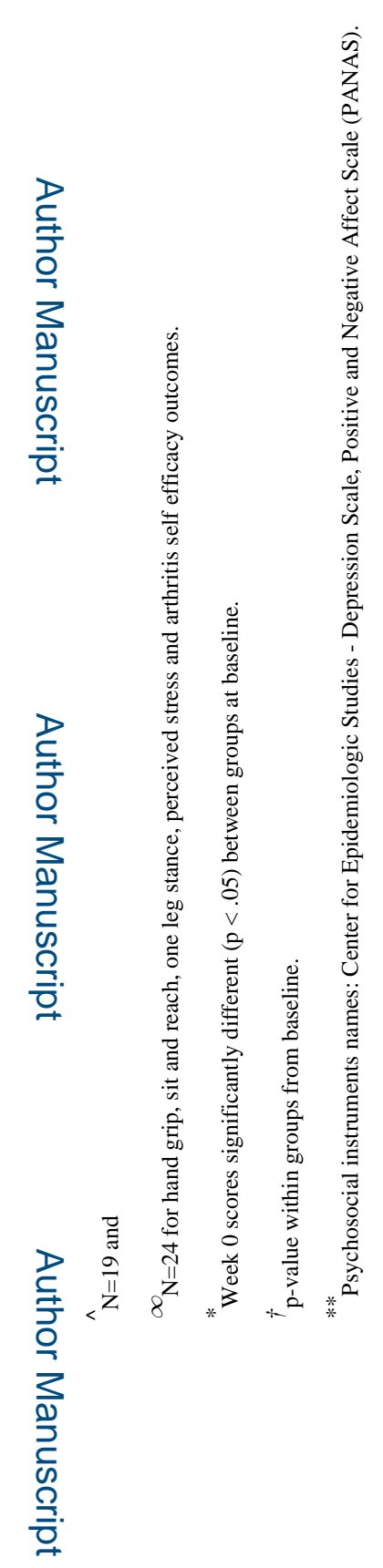

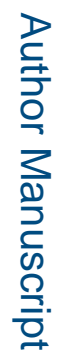

J Rheumatol. Author manuscript; available in PMC 2015 July 03. 


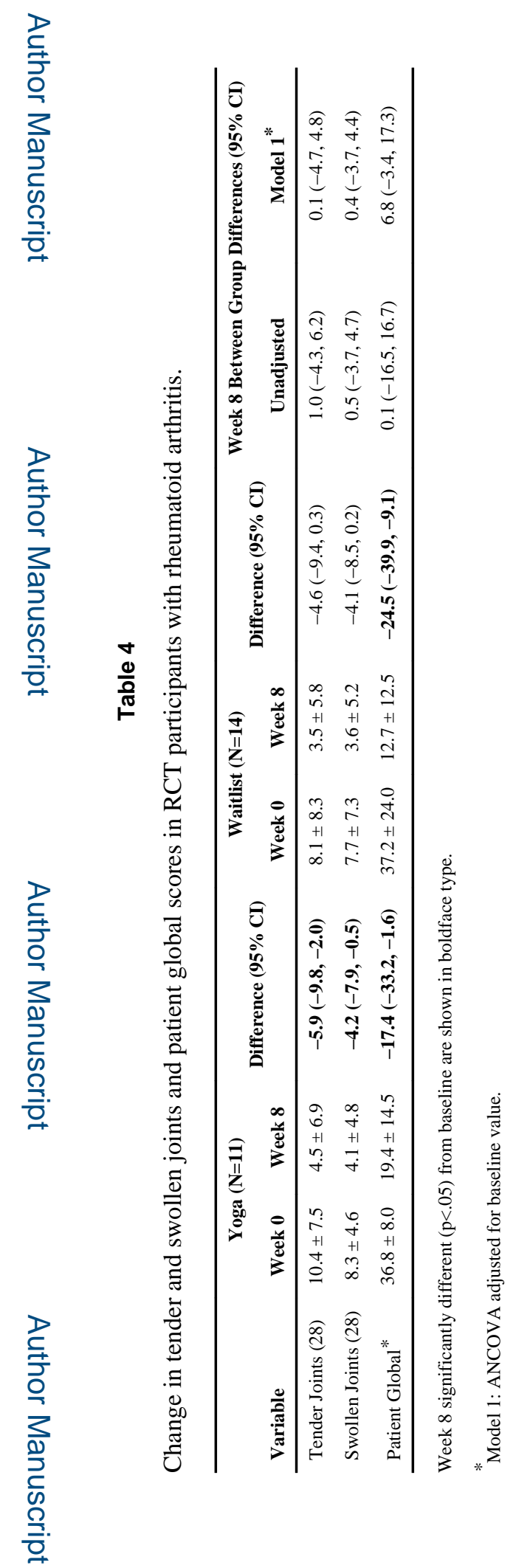

$J$ Rheumatol. Author manuscript; available in PMC 2015 July 03. 


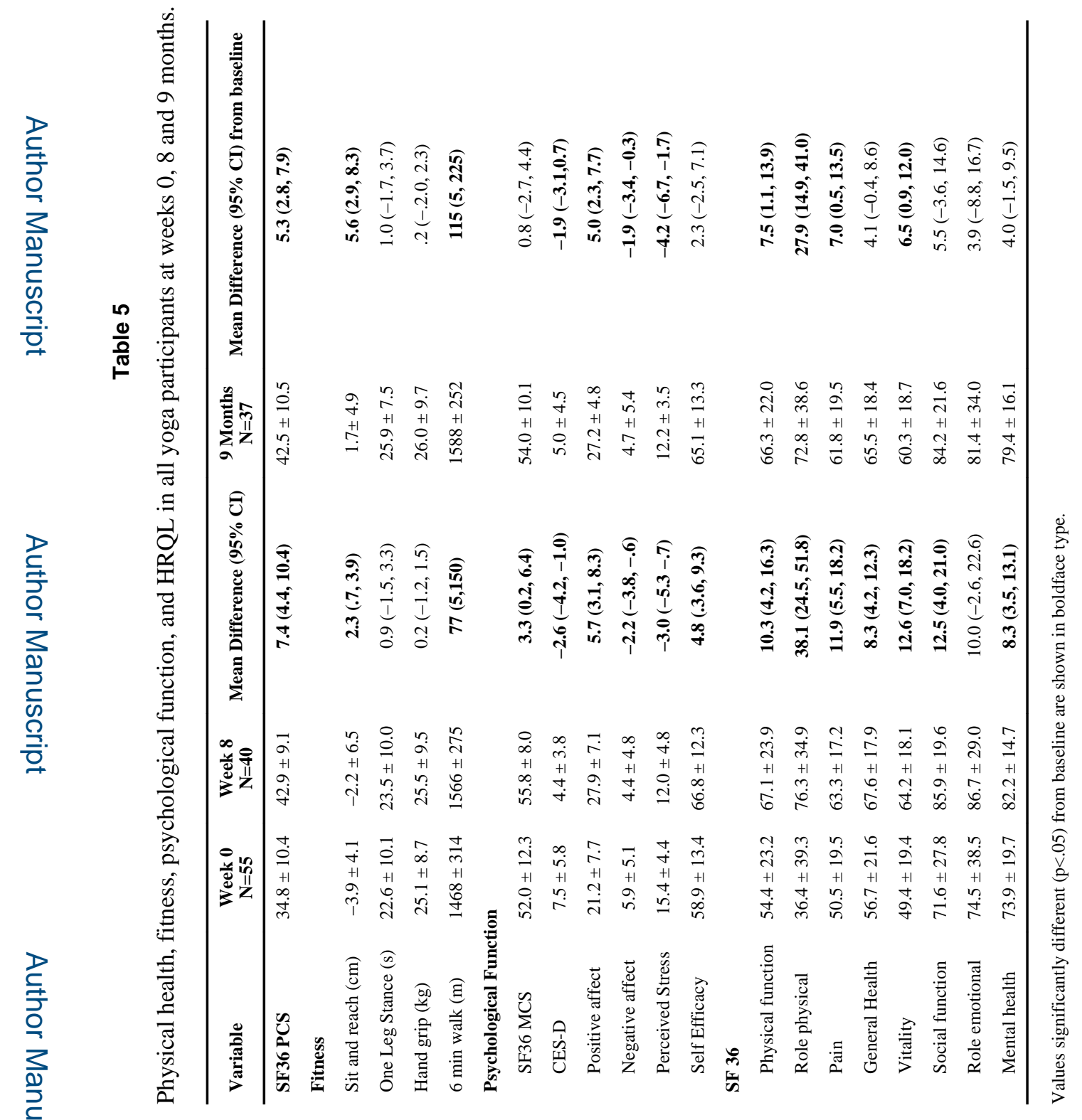

J Rheumatol. Author manuscript; available in PMC 2015 July 03. 